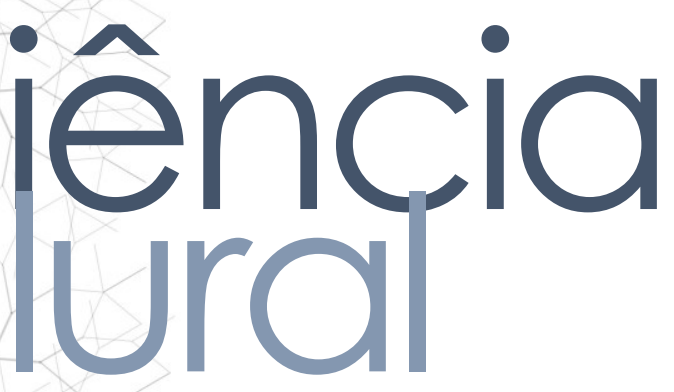

\title{
A UTILIZAÇÃO DA APRENDIZAGEM BASEADA EM PROBLEMA (ABP) NA FORMAÇÃO EM SAÚDE: UM RELATO DE EXPERIÊNCIA
}

The use of problem-based learning ( $p b l)$ in health training: a report of experience El uso del aprendizaje basado en problemas ( $p b l)$ en la formación en salud: un informe de experiencia

Isaac Newton Machado Bezerra • Graduado em Saúde Coletiva pela Universidade Federal do Rio Grande do Norte-UFRN • Residente em Atenção Básica pela Universidade Federal de Pernambuco- UFPE • E-mail: isaac.ufrn30@gmail.com

Jânio Luiz do Nascimento • Graduando do Curso de Enfermagem da UFRN • E-mail: janiolnascimento@gmail.com

Natalia Raiane Silva Vieira • Graduada em Saúde Coletiva • Enfermeira • Especialista em enfermagem do trabalho • E-mail: nataliaravieira@hotmail.com

Rafaela Priscila Carvalho da Silva • Graduanda do Curso de Saúde Coletiva da UFRN • E-mail: rafaelapriscila25@hotmail.com

Vinícius Costa Maia Monteiro • Secretaria de Estado da Saúde Pública-SESAP • Enfermeiro • pós-graduando em Saúde Coletiva-FAVENI • Professor do Instituto Técnico do Brasil e do Centro Educacional Técnico Profissional • E-mail:vinicius_catolico@hotmail.com

Zacarias Ramalho Silverio • Psicólogo • Especialista em Saúde Coletiva-UFRN • Docente na Faculdade Estácio - FATERN • E-mail: zak.psicologia@hotmail.com

Mariel Wágner Holanda Lima • Mestre em educação pela UFRN • Professor na Secretaria Estadual de Educação do Rio Grande do Norte • E-mail: marielwh@yahoo.com.br Maria da Conceição Lima Alves • Enfermeira • E-mail: malves@gmail.com Flávia Christiane de Azevedo Machado • Docente do Departamento de Saúde Coletiva da UFRN, Doutora em Saúde Coletiva-UFRN • E-mail: flavitamachado@yahoo.com.br Jônia Cybele Santos Lima • Docente do Departamento de Saúde Coletiva-UFRN • Mestre em Odontologia-Saúde Coletiva • E-mail: joniacybele@yahoo.com.br

Autor responsável pela correspondência • Isaac Newton Machado Bezerra

E-mail: isaac.ufrn30@gmail.com 


\section{ciêncíncia
pural}

\section{RESUMO}

Introdução: Nos últimos anos tem se evidenciado a necessidade de implementar novas metodologias no ensino, que consigam responder as mudanças tecnológicas, sociais e culturais. Neste cenário, as metodologias ativas vêm se destacando ao colocar o discente no centro do aprendizado, tornando-o parte integral e essencial desse processo. Dentre essas metodologias, está a Aprendizagem Baseada em Problemas. Essa metodologia busca o ensino a partir da solução de problemas, mediante análise em grupo, utilizando-se de conhecimentos previamente adquiridos, facilitando a apreensão do conhecimento e valorização do trabalho em equipe. Objetivo: Relatar a experiência de discentes de um curso da área da saúde no desenvolvimento de um caso e sua aplicação no formato de uma Aprendizagem Baseada em Problemas. Método: Estudo descritivo, tipo relato de experiência, onde se elabora o processo de construção desse tipo de aprendizagem e verificação da pertinência da estratégia proposta por discentes de um curso da saúde da Universidade Federal do Rio Grande do Norte na disciplina de Teoria Geral das Organizações. Resultados: Durante a criação e execução do caso, os discentes utilizaram diversas técnicas para construir ações de enfrentamento ao caso apresentado, como a técnica de Braisntorming, o Diagrama de Causa-Efeito, construção de critérios utilizando materiais do Ministério da Saúde, consultando profissionais do serviço em busca de estratégias viáveis para esses profissionais como forma de validação. Conclusões: $\mathrm{O}$ desenvolvimento de um caso utilizando as metodologias que integram a Aprendizagem Baseada em Problemas propiciou aos envolvidos a experimentação de um processo criativo, rico, centrado no conhecimento. A participação dos profissionais das redes de serviços de saúde foi fundamental em todas as fases, evidenciando sua potencialidade formadora indo desde a academia aos serviços de saúde com aplicabilidade e eficiência.

Palavras-Chave: Aprendizagem Baseada em Problemas; Práticas Interdisciplinares; Educação Superior.

\section{ABSTRACT}

Introduction: In recent years, the need to implement new teaching methodologies that can respond to technological, social and cultural changes has been highlighted. In this scenario, active methodologies have been standing out by placing the student at the center of learning, making it an integral and essential part of this process. Among these methodologies is Problem-Based Learning. This methodology seeks to teach from problem solving through group analysis, using previously acquired knowledge, facilitating the understanding of knowledge and the appreciation of teamwork. bjective: Report the experience of health course students in the development of a case and its application in the form of Problem-Based Learning. Methods: This is a descriptive study, type experience report, which describes the process of building roblem-Based Learning and verifying the relevance of the proposed strategy by udents of health courses of the Federal University of Rio Grande do Norte in the scipline of General Theory of Organizations. Results: During the creation and cution of the case, the students used various techniques to construct coping actions he case presented, such as the Braisntorming technique, the Cause-Effect Diagram, 
criteria construction using Ministry of Health materials, consulting professionals of the service in search for viable strategies for these professionals as a form of validation. Conclusions: The development of a case using the methodologies that integrate Problem-Based Learning has enabled those involved to experiment with a creative, rich, knowledge-centered process. The participation of professionals in the health service networks was essential in all phases, showing their training potential, ranging from the academy to health services with applicability and efficiency.

Keywords: Problem-Based Learning; Interdisciplinary Placement; Education, Higher.

\section{RESUMEN}

Introducción: en los últimos años ha sido necesario implementar nuevas metodologías de enseñanza que puedan responder a los cambios tecnológicos, sociales y culturales. En este escenario, las metodologías activas se han destacado al colocar al estudiante en el centro de aprendizaje, convirtiéndolo en una parte integral y esencial de este proceso. Entre estas metodologías se encuentra el Aprendizaje Basado en Problemas. Esta metodología busca la enseñanza desde la resolución de problemas hasta el análisis grupal, utilizando el conocimiento previamente adquirido, facilitando la comprensión del conocimiento y la apreciación del trabajo en equipo. Objetivo: Informar la experiencia de los estudiantes de cursos de salud en el desarrollo de un caso y su aplicación en el formato de Aprendizaje Basado en Problemas. Método: Este es un estudio descriptivo, informe de experiencia tipo, que describe el proceso de construcción de Aprendizaje Basado en Problemas y la verificación de la relevancia de la estrategia propuesta por estudiantes de cursos de salud en la Universidad Federal de Río Grande del Norte en la disciplina de Teoría General de Organizaciones. Resultados: Durante la creación y ejecución del caso, los estudiantes utilizaron diversas técnicas para construir acciones de afrontamiento al caso presentado, como la técnica de Braisntorming, el Diagrama de Causa-Efecto, la construcción de criterios utilizando materiales del Ministerio de Salud, la consulta de profesionales del servicio en busca de estrategias viables para estos profesionales como forma de validación. Conclusiones: El desarrollo de un caso utilizando las metodologías que integran el aprendizaje basado en problemas ha permitido a los involucrados experimentar con un proceso creativo, rico y centrado en el conocimiento. La participación de profesionales en las redes de servicios de salud fue esencial en todas las fases, mostrando su potencial de capacitación, desde la academia hasta los servicios de salud con aplicabilidad y eficiencia.

Palabras clave: Aprendizaje Basado en Problemas; Prácticas Interdisciplinarias; Educación Superior. 


\section{Introdução}

As Instituições de Ensino Superior historicamente balizaram seu modo de operacionalizar o processo de ensino-aprendizagem com base no relatório Flexner Medical Education in the United States and Canada - A Report to the Carnegie Foundation for the Advancement of Teaching, publicado em $1910^{1}$. Todavia, o que era adequado aquele contexto histórico, já não é para a atualidade. Hoje novas tecnologias, pesquisas pedagógicas acerca do aprendizado e necessidade de relações horizontais na relação docente-discente, coloca-se a veemência de romper o paradigma Flexneriano vigente, simbolizado pela conformação das salas de aula onde o docente está a frente dos discentes como o objeto central do processo ${ }^{2,3}$.

Por conseguinte, metodologias pautadas na centralidade do discente, no estímulo a seu protagonismo e no papel do docente como facilitador mostram-se mais coerentes às necessidades sociais vigentes ${ }^{4}$.

Dentre essas metodologias, está a Aprendizagem Baseada em Problemas (ABP). A ABP busca o ensino a partir da solução de problemas, mediante análise em grupo, utilizando-se de conhecimentos previamente adquiridos, facilitando a apreensão do conhecimento e a valorização do trabalho em equipe. Não obstante, a ABP vem sendo amplamente utilizada nos cursos da área da saúde 5,6 .

A ABP tem sua primeira inserção no ensino da saúde na Universidade de McMaster no Canadá em 1969, trazendo uma proposta pedagógica sólida, baseando o ensino na solução de problemas reais e fictícios, buscando a integração entre os discentes e incentivando o pensamento crítico-reflexivo acerca das temáticas abordadas ${ }^{7}$.

Desta forma, a ABP integrante da problematização, vai ao encontro do preconizado nas Diretrizes Curriculares Nacionais (DCN) para a formação em saúde, buscando viabilizar o trabalho de habilidades atitudinais (análise crítica, reflexiva) e elacionais (trabalho em equipes) ${ }^{8}$.

Mediante a potencialidade da $\mathrm{ABP}$, esse trabalho objetiva relatar a experiência discentes de cursos da área da saúde no desenvolvimento de um caso e sua 
aplicação no formato de uma ABP, na disciplina de Teoria Geral das Organizações, oferecida pelo Departamento de Administração da Universidade Federal do Rio Grande do Norte-UFRN ao curso de Graduação em Saúde Coletiva.

\section{Método}

Trata-se de estudo descritivo, tipo relato de experiência, onde se descreve o processo de construção da $\mathrm{ABP}$ e verificação da pertinência da estratégia proposta. $\mathrm{O}$ intuito é apontar os resultados satisfatórios obtidos, bem como as fragilidades identificadas para viabilizar a reprodutibilidade da experiência.

\section{Contexto}

No primeiro semestre de 2018, foi solicitada, durante a disciplina de Teoria Geral das Organizações ofertada aos discentes da Graduação em Saúde Coletiva da Universidade Federal do Rio Grande do Norte-UFRN, a construção de um caso no formato de uma ABP. No caso, esta construção constituiu-se em requisito para obtenção de nota parcial da terceira unidade. Tal atividade deveria a ser desenvolvida em grupo.

Desta forma, a construção do caso envolveu as seguintes etapas:

- Brainstorming para selecionar a temática-alvo, bem como a estratégia de abordála.

- Apresentação da estratégia construída a técnicos da Secretaria Municipal de Saúde (SMS) de Natal atuantes como informantes-chave e viabilizando uma validação de conteúdo.

- Oficina para verificação da pertinência do caso construído (análise da pertinência da estratégia por meio da vivência da mesma pelo grupo idealizador).

Para o desenvolvimento das etapas do Brainstorming e da oficina, o grupo, composto por seis integrantes, foi dividido em dois. Um grupo ficou responsável pelo esenvolvimento do caso e da estratégia de como conduzi-lo e o outro grupo ivenciou a oficina para verificar a pertinência da estratégia. 
O Brainstorming ocorreu em duas reuniões com duração de 1 hora cada. $\mathrm{Na}$ primeira reunião, o objetivo foi à seleção da temática a partir de critérios: relevância social, pertinência para as ações desenvolvidas no contexto do SUS, pertinência do tema selecionado frente o perfil epidemiológico vigente, relevância para o campo da saúde coletiva e o seu potencial crítico-reflexivo da temática para suscitar trabalhar habilidades cognitivas, atitudinais e relacionais. Desta reunião inicial, participaram todos os integrantes do grupo.

Definida a temática, no caso o rastreamento e prevenção do Vírus do Papiloma Humano $(H P V)$, a segundo reunião teve por foco a definição da estratégia. Então, procedeu-se o processo de divisão de atividades no grupo, iniciando-se, neste momento, a construção do caso problema com participação de alguns membros do grupo. Tal construção ocorreu em dois encontros, uma vez que foi preciso desenvolver um texto adequado aos critérios estabelecidos pelo grupo, inserir elementos pertinentes que viabilizassem a análise crítica-reflexiva do caso conforme os preceitos da APB. O grupo deliberou que a análise do caso deveria ser realizada a partir da ótica de um Gerente de uma Unidade de Saúde da Família (USF). Isto porque, um dos objetivos da graduação em saúde coletiva é viabilizar a formação de sanitaristas com habilidades para o exercício da gerência e gestão9 ${ }^{9}$ O texto deveria apresentar clareza, coerência e coesão, uma vez que a partir da interpretação do mesmo iriam ser propostas soluções ao caso problema. Tais soluções deveriam ser baseadas em evidências, construídas com auxílio de ferramentas utilizadas no planejamento estratégico situacional (Diagrama de Causa-Efeito -Ishikawa- e a Matriz GUT de Priorização) e coerente às demandas descritas no caso.

Definido o caso e como este deveria ser desenvolvido, foi feita a apresentação a técnicos da SMS Natal para que verificassem a pertinência e fizessem alterações, caso necessário, durante o terceiro encontro do grupo. No caso, foi colocada a pertinência.

Assim, iniciou-se a etapa de verificação da pertinência da estratégia proposta través de uma oficina ocorrida em uma das quatro reuniões do grupo. Desta forma, grupo idealizador se reuniu e acompanhou o processo de verificação da pertinência estratégia proposta mediante a vivência da ABP por outros integrantes do grupo, 


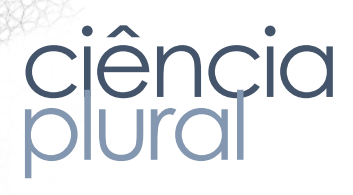

não envolvidos na idealização e desenvolvimento do caso. Desta forma, a análise do caso se deu a partir da construção de um diagrama de causa-efeito, objetivando um diagnóstico situacional e a verificação da cadeia causal envolvida na problemática descrita no caso. Em seguida, partiu-se para a priorização do problema, dentre os identificados na cadeia causal construída com auxílio do diagrama causa-efeito, com o auxílio da matriz GUT de priorização. Priorizado o problema, iniciou-se a proposição de um plano operativo considerando a análise de viabilidade do plano, bem como o momento de avaliação dos resultados alcançados pelo plano mediado pelo uso de indicadores.

O Diagrama de Ishikawa, também conhecido como diagrama de causa-efeito ou espinha de peixe, é uma importante ferramenta utilizada para investigação de um problema, descrevendo seus agentes causadores e variáveis envolvidas, de acordo com suas causas e categorias. Os fatores (causas) são identificados via brainstorming ou brainwriting. Esses fatores identificados no processo de geração de ideias devem são agrupados por similaridade nas categorias previamente definidas (espinhas principais). Desta forma, deve-se analisar o diagrama dando maior ênfase aos fatores que aparecem em mais de uma categoria ${ }^{10}$.

Por sua vez, a Matriz GUT visa definir prioridades por meio da utilização de critérios predefinidos tais como: gravidade, urgência e tendência do fenômeno ao qual se aplica (11). Para tanto, a matriz utiliza a seguinte sistemática: 1-Classifica as prioridades através de critério de pontuação de 1 a 5; 2- Aqueles problemas com maiores pontuações deverão ser combatidos prioritariamente para melhoria do processo de trabalho; 3-A maior pontuação é 125 (5x5x5 - notas máximas atribuídas aos 03 critérios), enquanto a menor é (1x 1x1). Tais ferramentas são características do Planejamento Estratégico Situacional (PES) que pressupõe constante adaptação para cada situação concreta onde é aplicado, dividindo-se em quatro momentos, explicativo; descrição do problema, afastando-se nesse momento ambiguidades, normativo; elaboração das ações para o enfrentamento dos nós críticos, estratégico: álise da viabilidade do plano, com a definição de responsáveis e estabelecimento de zos iniciais e tático-operacional; momento dedicado à execução do plano, avaliação estação de contas ${ }^{12}$. 
Assim, os resultados deste relato referem-se a este processo de construção e verificação da pertinência da estratégia construída para uma atividade baseada no método da ABP, perpassando os momentos do Planejamento Estratégico.

\section{Resultados}

\section{O Caso}

"O falecimento de uma jovem de 26 anos, mãe de dois filhos, após anos de luta contra um câncer de colo do útero, descoberto há três anos em estado avançado, levantou novos questionamentos.

A investigação do óbito realizada pela Vigilância Epidemiológica municipal de Várzea Feliz evidenciou que a jovem era frequentadora assídua da Unidade de Saúde da Família (USF) de seu bairro e antes de ser diagnosticada com a doença havia reportada queixas, por diversas vezes, aos profissionais da Equipe de Saúde da Família (ESF) que a atendia. Neste sentido, relatos de amigos e parentes afirmam e confiram que a jovem queixava-se constantemente de dores ao urinar, aparecimento constante de sangue em sua urina, corrimento sanguinolento vaginal, além de fortes dores nas pernas, quadris e costas. Além disso, a perda de peso era notória. Todavia, mesmo diante dos sintomas, o diagnóstico só foi obtido quando foi encaminhada para a capital do estado e foi possível realizar uma série de exames. Ainda, segundo relatos de familiares, durante esse período a usuária recebeu diferentes diagnósticos dos médicos da cidade onde mora, incluindo anemia e virose. Sendo assim, diversos tratamentos foram indicados, mas nenhum conseguiu resolver o problema. Apesar de apresentar momentos de melhora por uns dias, logo voltava a apresentar os mesmos sintomas. A irmã da jovem relata que ela não foi a primeira da família diagnosticado com esse tipo de neoplasia. A mãe, a tia (paterna) e uma prima (paterna) receberam o mesmo diagnóstico.

O município apresenta dificuldades orçamentárias e também relacionadas à oferta e serviços: ausência de insumos básicos, Recursos Humanos insuficientes, Equipe Estratégia Saúde da Família incompleta (desassistida de médico) o que tem icultado o trabalho das unidades. 
Por ser um município de pequeno porte, possuindo 4.800 habitantes, serviços de média e alta-complexidade não são oferecidos à população, que necessitam se deslocar até a capital do estado, localizada a $210 \mathrm{~km}$ para ter acesso a esses serviços".

Fonte: autores (2018)

As conversas desenvolvidas com os profissionais do serviço serviram como base para a organização das estratégias e orientação para definição de um ponto de partida.

O grupo de análise construiu um quadro de metas utilizando o brainstorming (Quadro 1), e um diagrama de Ishikawa (Figura 1) apontando as possíveis causas relacionadas aos problemas descritos e elaborou critérios para as ações utilizando informações retiradas de documento oficial do Ministério da Saúde ${ }^{13}$ que abordam a temática do HPV (Quadro 2).

Quadro 1: Quadro de metas após discussão.

A. Realizar preventivo anualmente em toda mulher na faixa etária de 25 a 64 anos atendida nas USF;

B. Realizar a vacinação de $100 \%$ do público alvo;

C. Realizar vacinação de $100 \%$ do grupo prioritário;

D. Realizar acompanhamento da população com vacinação iniciada para realização do esquema correto de vacinação

E. Dar seguimento correto aos casos diagnosticados de lesões

Fonte: Autores, 2018.

Durante a análise do caso, além das consultas ao Manual de vacinas do Ministério da Saúde, profissionais do serviço foram consultados para ajudarem na construção de um método de enfrentamento que pudesse coadunar ações de profissionais da Atenção Primária à Saúde e Média Complexidade. 
Figura 1: Diagrama de Ishikawa elaborado de acordo com a definição do problema prioritário identificado pelo grupo de análise do ABP.

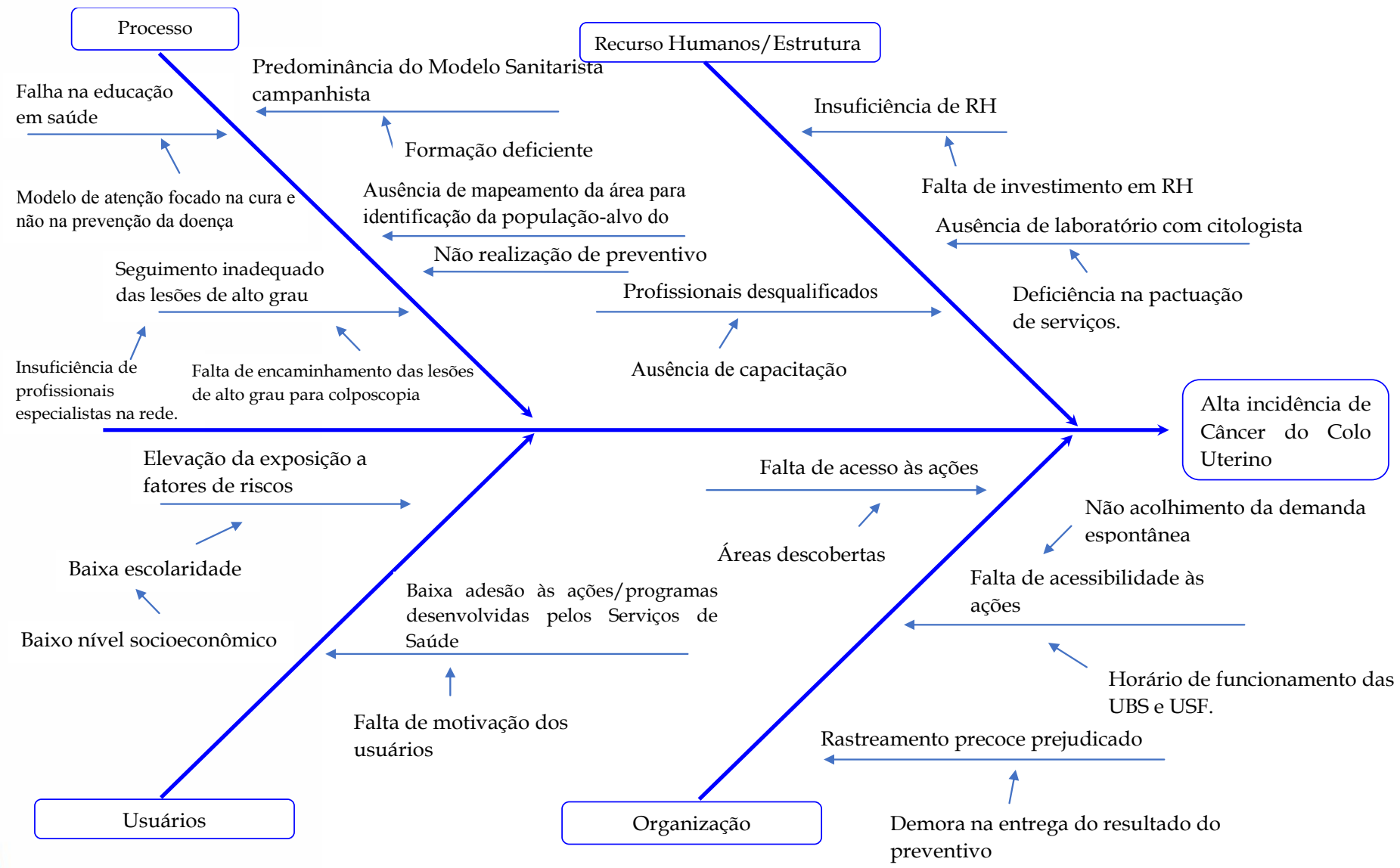

Fonte: Autores, 2018.

\section{Quadro 2: Critérios, exceções e esclarecimentos com base nas orientações do}

Manual de Normas e Procedimentos para Vacinação, 2014 do Ministério da Saúde.

\begin{tabular}{|c|c|c|}
\hline Definição do critério. & Exceções & Esclarecimentos \\
\hline $\begin{array}{l}\text { Toda mulher na faixa etárias de } 25 \text { a } \\
64 \text { anos atendidas nas USF deve } \\
\text { realizar preventivo anualmente. }\end{array}$ & $\begin{array}{l}\text { 1. } \quad \text { Mulheres com } \\
\text { lesão de baixo grau. } \\
\text { 2. } \text { HPV positivo. } \\
\text { 3. } \quad \text { HIV positivo. }\end{array}$ & $\begin{array}{l}\text { Mulheres com lesão de baixo nível ou HPV } \\
\text { positivo devem realizar o exame semestralmente } \\
\text { para acompanhamento da lesão }{ }^{13} \\
\text { Mulheres com HIV positivo devem realizar o } \\
\text { exame semestralmente no primeiro ano, e se } \\
\text { resultados normais (ausência de lesão) manter } \\
\text { segmento anual }{ }^{13} \text {. }\end{array}$ \\
\hline
\end{tabular}




\begin{tabular}{|c|c|c|}
\hline $\begin{array}{l}\text { Toda menina entre } 9 \text { e } 14 \text { anos e } \\
\text { meninos de } 12 \text { a } 13 \text { anos devem ser } \\
\text { vacinados contra HPV. }\end{array}$ & $\begin{array}{l}\text { 1. Gestantes. } \\
\text { 2. Pessoas com } \\
\text { Hipersensibilidade ao } \\
\text { princípio ativo da } \\
\text { vacina. }\end{array}$ & $\begin{array}{l}\text { A vacina não é recomendada para gestantes e } \\
\text { pessoas com Hipersensibilidade aos princípios } \\
\text { ativos da vacina, pois ainda não houve teste da } \\
\text { vacina nessa população específica }{ }^{13} \text {. }\end{array}$ \\
\hline $\begin{array}{l}\text { Toda pessoa de grupos prioritários* } \\
\text { deve ser vacinada contra HPV. }\end{array}$ & $\begin{array}{l}\text { 1. Gestantes. } \\
2 . \quad \text { Pessoas com } \\
\text { Hipersensibilidade ao } \\
\text { princípio ativo da } \\
\text { vacina. }\end{array}$ & $\begin{array}{l}\text { A vacina não é recomendada para gestantes e } \\
\text { pessoas com Hipersensibilidade aos princípios } \\
\text { ativos da vacina, pois ainda não houve teste da } \\
\text { vacina nessa população específica }{ }^{13} \text {. } \\
\text { *Grupos prioritários para a vacinação do HPV são: } \\
\text { pessoa vivendo com HIV e pessoas em tratamento } \\
\text { de Neoplasias de } 9 \text { a } 26 \text { anos de ambos os sexos }{ }^{13} \text {. }\end{array}$ \\
\hline $\begin{array}{l}\text { Toda população com vacinação } \\
\text { iniciada contra HPV deve ser } \\
\text { acompanhada para realização do } \\
\text { esquema correto* de vacinação. }\end{array}$ & $\begin{array}{l}\text { 1. Gestantes. } \\
2 . \quad \text { Pessoas com } \\
\text { Hipersensibilidade ao } \\
\text { princípio ativo da } \\
\text { vacina. }\end{array}$ & $\begin{array}{l}\text { A vacina não é recomendada para gestantes e } \\
\text { pessoas com Hipersensibilidade aos princípios } \\
\text { ativos da vacina, pois ainda não houve teste da } \\
\text { vacina nessa população específica }{ }^{13} \text {. } \\
{ }^{*} \text { Esquema correto: } 2 \text { doses para população sem } \\
\text { grau de vulnerabilidade e } 3 \text { doses para grupos } \\
\text { vulneráveis, HIV positivo e em tratamento de } \\
\text { Neoplasias }{ }^{13} \text {. }\end{array}$ \\
\hline $\begin{array}{l}\text { Todos os casos diagnosticados de } \\
\text { lesões devem ser encaminhados } \\
\text { (seguimento adequado*) de forma } \\
\text { adequada para tratamento. }\end{array}$ & - & $\begin{array}{l}\text { *Segmento adequado: Após diagnostico de lesão } \\
\text { existente, o usuário (a) deve ser encaminhado para } \\
\text { médico especialista para início de tratamento }{ }^{13} \text {. }\end{array}$ \\
\hline
\end{tabular}

Fonte: Autores, 2018.

\section{Discussão}

A utilização de metodologias ativas no ensino da saúde é algo bem difundido e vem ganhando legitimidade crescente a cada ano. Porém a metodologia tradicional de aprendizado baseado na aplicação de avaliações objetivas e discursivas ainda é predominante no sistema de ensino brasileiro $5,14,15$.

Todavia, esforços devem ser continuamente implementados para ruptura da ducação tradicional. A aplicação da metodologia da ABP estimula o pensamento tico, o trabalho em equipe e a análise das capacidades do grupo, impulsionando o cente a recorrer a suas habilidades e conhecimentos prévios, buscar novas 


\section{ciência
piural}

informações e ressignificar elaborações sobres os fenômenos estudados, construindo uma aprendizagem ativa, contextualizada a realidade $16,17$.

Experiência exitosa com a ABP tem sido divulgada, aumentando sua visibilidade e utilização, tanto nos cursos de graduação da saúde 5,7,18 e de outras áreas do conhecimento como Engenharia da Produção 19, Arquitetura e Urbanismo 20, e Administração ${ }^{21}$.

O emprego desse método como forma de avaliação traz significativas contribuições, não apenas no campo acadêmico, mas também no pessoal e profissional do estudante ${ }^{22}$. Isto porque a $\mathrm{ABP}$ envolve o processo de negociação de diferentes interesses do grupo, deliberação de consenso, divisão de atividades do grupo com base nas habilidades de cada membro para ser atingido o objetivo e, neste processo, comporta habilidades de liderança.

A ABP, assim como qualquer método, necessita de uma avaliação a fim de uma melhoria contínua de sua aplicabilidade. Existem cenários diversos de aprendizado e cada um tem suas peculiaridades ${ }^{23}$. Entretanto, independente dos contextos há recursos essenciais para a ABP: instrutores conhecedores do método para instigar o espírito crítico e investigativo dos participantes; parceria com profissionais atuantes nos serviços de saúde para auxiliarem na avaliação da pertinência dos casos, bem como na viabilidade das estratégias de intervenção aventadas pelos participantes.

No estudo vigente identificamos fragilidades relacionadas a não captação das percepções dos participantes do ABP acerca das contribuições do método para seu aprendizado, bem como a não inclusão de informações no caso necessárias a análise de viabilidade do plano operativo proposto.

Em relação a não captação das percepções, optou-se verificar a adequação da proposta construída e pertinência das ferramentas utilizadas no processo; o Ishikawa e matriz GUT, bem como o plano operativo proposto. Quanto a não inclusão de nformações, ao observar as estratégias propostas de vacinar todas as meninas e ninos na idade alvo da vacina de prevenção ao HPV, seria necessário o quantitativo ta população para análise de viabilidade do plano. Inclusive, em relação ao plano, ve ausência de informações de quais ações a serem desenvolvidas para contemplar 
os critérios propostos e assim, atingir as metas discriminadas. Com isto, o momento estratégico do planejamento poderia ter sido mais detalhado pelos participantes.

Por sua vez, analisando as potencialidades da experiência descrita, destaca-se a proatividade dos discentes que desenvolveram a ABP. Ressalte-se que o relato indica o desfecho de uma atividade avaliativa de uma Instituição de Ensino Superior e inferese sobre a concretização da articulação da tríade ensino-extensão-pesquisa. No caso, a extensão ocorre com a inclusão de profissionais da Secretaria de Saúde atuantes como informantes-chave. Ao considerar a dialética do processo ensino-aprendizagem podese inferir uma contribuição social com os serviços de saúde. Por sua vez, o desenvolvimento deste relato materializa a perspectiva da pesquisa.

Para além desses pontos, a experiência da ABP foi positiva, uma vez que houve adequada condução do método e o Diagrama de Ishikawa produzido reflete o processo de análise crítica dos discentes participantes. O momento explicativo do planejamento onde é realizada uma análise da realidade apresentada, para identificar problemas, priorizar e discutir a cadeia causal relacionada a este problema exige o exercício de importantes habilidades intelectuais e técnicas. Assim, há uma mobilização de teoria-prática aplicada, essencial a aprendizagem significativa e libertadora.

A observação detalhada do Diagrama de Ishikawa permite a inferência do quão produtivo foi o processo de discussão do brainstorming. No caso, as causas estão relacionadas a diferentes níveis de complexidade, desde questões mais distais como predominância do modelo sanitarista campanhista as mais proximais, como ausência de capacitação observando o grupo Recursos Humano/Estrutura, fator apontado pela literatura como importante na qualidade dos serviços ofertados ${ }^{24}$.

Quanto aos grupos presentes no Ishikawa (Processo, usuários, organização, recursos) há uma evidente apreensão sobre os diferentes grupos de variáveis que influenciam nas problemáticas que acometem os serviços de saúde. Deste modo, a erramenta construída per si denota a efetividade da ABP na presente experiência.

Por fim, quanto ao momento normativo do planejamento onde é colocado a uação desejada, esta foi claramente definida analisando as metas do quadro 1. 


\section{ciência
piural}

Assim, indica-se a reprodução da experiência diante dos bons resultados apresentados, sobretudo, buscando implementar as questões aqui identificadas como fragilidades e incluir nas discussões a orientação familiar. Desta forma, deve-se fornecer mais informações sobre o município, estimular o desenvolvimento de um plano operativo baseado no método do $5 \mathrm{~W} 2 \mathrm{H}$.

\section{Conclusões}

O desenvolvimento de um caso utilizando as metodologias que integram a ABP propiciou aos envolvidos a experimentação de um processo criativo, rico e centrado no conhecimento partilhado pelos membros da equipe, ajudando a despertar um olhar mais aguçado sobre o problema e refletindo de forma construtiva sobre como é enfrentar as dificuldades relacionadas a execuções de ações nos serviços de saúde.

A participação de profissionais dos serviços do SUS nas diversas fases de elaboração e execução do ABP se mostrou de extrema importância, pois a partir de seus olhares a experiência pode ser mais balizada pelas reais dificuldades enfrentadas no dia-a-dia dos serviços públicos.

No tocante a interação com serviço, para o ensino ficou evidenciado o diferencial no processo formativo para a saúde, com as inter-relações e as interlocuções que surgem do estreitamento dessa relação. Assim, com as atividades vivenciadas nos cenários de práticas pôde-se mobilizar a interação da teoria e a pratica junto aos processos de trabalho na saúde.

Diante desse contexto, fica evidente que a ABP possui um amplo campo de utilização, podendo ser utilizado não somente para ensino de futuros profissionais, mas nos próprios serviços de saúde como metodologia de educação continuada para seus profissionais e de educação popular em saúde para seus usuários.

\section{eferências}

Flexner A. Medical education in the United States and Canada: A report to the rnegie Foundation for the Advancement of Teaching - Bulletin Number 4. New k: Carnegie Foundation for the Advancement of Teaching. 1910. 
2. Gomes MP, Ribeiro VM, Monteiro DM, Leher EMT, Louzada R de CR. O uso de metodologias ativas no ensino de graduação nas ciências sociais e da saúde:

avaliação dos estudantes. Ciência Educ [Internet]. 2010;16(1):181-98. Disponível em: http:/ / www.scielo.br/scielo.php?script=sci_arttext\&pid=S1516-

73132010000100011\&lng=pt\&tlng=pt

3. Fujita JALM, Mecena EH, Carmona EV, Shimo AKK. Uso da metodologia da problematização com o Arco de Maguerez no ensino sobre brinquedo terapêutico. Rev Port Educ [Internet]. 2016;29(1):229. Disponível em:

http://revistas.rcaap.pt/rpe/article/view/rpe.5966

4. $\quad$ Freire P. Pedagogia do oprimido. 50. ed. São Paulo: Paz e Terra, 2011.

5. Souza SC, Dourado L. aprendizagem baseada em problemas (abp): um método de aprendizagem inovador para o ensino educativo. HOLOS [Internet]. 2015 1;5:182. Disponível em:

http://www2.ifrn.edu.br/ojs/index.php/HOLOS/article/view/2880

6. Bergman EM, de Bruin AB, Herrler A, Verheijen IW, Scherpbier AJ, van der Vleuten $\mathrm{CP}$. Students' perceptions of anatomy across the undergraduate problembased learning medical curriculum: a phenomenographical study. BMC Med Educ [Internet]. 2013;13(1):152. Disponível em:

https://bmcmededuc.biomedcentral.com/articles/10.1186/1472-6920-13-152

7. Borges MDC, Chachá SGF, Quintana SM, Freitas LCC, Rodrigues MLV. Aprendizado baseado em problemas. Med (Ribeirao Preto Online) [Internet]. 2014;47(3):301. Disponível em:

http://www.revistas.usp.br/rmrp/article/view/86619

8. Mello CCB, Alves RO, Lemos SMA. Metodologias de ensino e formação na área da saúde: revisão de literatura. Rev CEFAC [Internet]. 2014 ;16(6):2015-28. Disponível em: http://www.scielo.br/scielo.php?script=sci_arttext\&pid=S1516$18462014000602015 \& \operatorname{lng}=$ pt\&tlng=pt

9. Brasil. Universidade Federal do Rio Grande do Norte. Projeto Pedagógico do Curso de Saúde Coletiva. Natal, 2018. Disponível em:

https://sigaa.ufrn.br/sigaa/public/curso/ppp.jsf?lc=pt_BR\&amp;id=725242; Acesso em: 30 abr 2018.

Wong KC, Woo KZ, Woo KH. Ishikawa Diagram. In. O’Donohue W, laragakis A. editores. Quality Improvement in Behavioral Health. Springer; 2016. p. $19-32$.

Fáveri R, Silva A. Método GUT aplicado à gestão de risco de desastres: uma amenta de auxílio para hierarquização de riscos. Revista Ordem Pública 
[Internet]. 2016;9(1):93-107 Disponível em: https:/ / rop.emnuvens.com.br/rop/article/view/112/105

12. Silva AK, Sousa JP, Rodrigues W, Cançado AC. Planejamento Estratégico Situacional - PES: uma análise bibliométrica da produção científica brasileira. Rev do Serviço Público [Internet]. 2017;68(2):365-88. Disponível em:

https://revista.enap.gov.br/index.php/RSP/article/view/1269

13. Brasil. Ministério da Saúde. Secretaria de Vigilância em Saúde. Departamento de Vigilância das Doenças Transmissíveis. Manual de Normas e Procedimentos para Vacinação. 1a ed. Brasília: DF. Ministério da Saúde; 2014.

14. Farias PAM, Martin ALAR, Cristo CS. Aprendizagem Ativa na Educação em Saúde: Percurso Histórico e Aplicações. Rev Bras Educ Med [Internet]. 2015;39(1):143-50. Disponível em:

http:/ / www.scielo.br/scielo.php?script=sci_arttext\&pid=S010055022015000100143\&lng=pt\&tlng=pt

15. Pivetta HMF, Vogt MSL, Badaró AFV. Metodologia do ensino superior: uma experiência na pós-graduação lato sensu em fisioterapia. Cad. Educ. Saúde Fisioter [Internet]. 2015;1(2):85-94. Disponível em:

http://revista.redeunida.org.br/ojs/index.php/cadernos-educacao-saudefisioterapia/article/view/340

16. Silva ES, Dias BJC, Souza JLM, Lima MS. Aprendizagem baseada em problema aplicada no ensino de urgência e emergência na enfermagem: um relato de experiência. Brazilian J Heal Rev [Internet]. 2019;2(4):2525-9. Disponível em: http://www.brjd.com.br/index.php/BJHR/article/view/1831/1801

17. Leon LB de, Onófrio F de Q. Aprendizagem Baseada em Problemas na Graduação Médica - Uma Revisão da Literatura Atual. Rev Bras Educ Med [Internet]. 2015;39(4):614-9. Disponível em: http://dx.doi.org/10.1590/198152712015v39n4e01282014

18. Shankar PR, Nandy A, Balasubramanium R, Chakravarty S. Small group effectiveness in a Caribbean medical school's problem-based learning sessions. J Educ Eval Health Prof [Internet]. 2014;24;11:5. Disponível em: http://jeehp.org/DOIx.php?id=10.3352/jeehp.2014.11.5

19. Pinto CP, Scheidegger APG, Gaudêncio JHD, Turrioni JB. Planejamento, ondução e análise do método de avaliação de uma disciplina do curso de genharia de produção fundamentada na aprendizagem baseada em problemas.

Rev Produção Online [Internet]. 2015;15(2):671-695. Disponível em:

ittp:// producaoonline.org.br/rpo/article/view/1936 
20. Ferreira CL, Flório W, Iaralham LH. Os métodos educacionais para formação de profissionais reflexivos em Arquitetura e Urbanismo: ABP, AOP e ABD. Rev Intern Formación Profes. Adul y Comunidad [Internet] 2015;2:52-58. Disponível em: ttps://journals.epistemopolis.org/index.php/adultoscomunidad/article/view/956 $/ 520$

21. Guedes KL, Andrade ROB, Nicolini AM. A avaliação de estudantes e professores de administração sobre a experiência com a aprendizagem baseada em problemas. Adm Ensino e Pesqui [Internet]. 2015;16(1):71. Disponível em:

http://raep.emnuvens.com.br/raep/article/view/201

22. Rocha JS, Dias GF, Campanha NH, Baldani MH. O uso da aprendizagem baseada em problemas na Odontologia: uma revisão crítica da literatura. Rev da ABENO [Internet]. 2016;16(1):25-38. Disponível em:

https://revabeno.emnuvens.com.br/revabeno/article/view/231

23. Dornelas R, Silva K da, César CPHAR, Oliveira-Barreto AC de, GuedesGranzotti RB. Avaliação da Situação-Problema por tutores e discentes em um curso de graduação vivenciado em metodologias ativas. Rev Ibero-Americana Estud em Educ [Internet]. 2016;11(1):245-57. Disponível em:

http:/ / seer.fclar.unesp.br/iberoamericana/article/view/7613/5711

24. Silva TVS, Santos KMR, Ferreira TLS, Andrade FB. Avaliação do atributo orientação familiar na saúde da criança. Rev. Ciênc. Plur. [Internet]. 2019;5(2):1-15.

Disponível em: https:/ / periodicos.ufrn.br/rcp/article/view/16306 